\title{
O Ensino de Gestão de Antimicrobianos em Escola Médica do Rio de Janeiro
}

\author{
Teaching Antimicrobial Stewardship at a Rio \\ de Janeiro Medical School
}

André Ricardo Araujo da Silvale

Igor Melo de Almeida

Rafael Carvalho Sacre

Cristina Letícia Passos de Souzal

\section{PALAVRAS-CHAVE}

- Programas de Gestão de Antimicrobianos.

- Currículo.

- Medicina.
I Universidade Federal Fluminense, Niterói, Rio de Janeiro, Brasil.

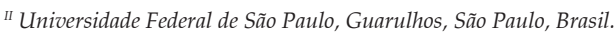

\section{RESUMO}

Introdução: Programas de gestão de antimicrobianos (PGA) são ferramentas importantes para minimizar o problema global da resistência antimicrobiana. As bases destes programas devem começar idealmente na graduação médica. Objetivo: Identificar a apresentação dos conceitos dos PGA no currículo de uma escola médica. Material e métodos: Estudo transversal realizado com docentes da Universidade Federal Fluminense (UFF). O critério de inclusão utilizado foi ministrar conteúdos relativos a PGA para o curso de Medicina. Foram mensurados apresentação de componentes-chave dos PGA, carga horária destinada, metodologias de ensino e avaliações empregadas. Resultados: Estudo realizado entre dezembro de 2017 e janeiro de 2018, sendo incluídos 6 dos 329 (1,8\%) docentes que ministravam aulas para o curso de Medicina. A carga horária destinada à apresentação dos PGA totalizou 83 horas, com mediana de 3 h/docente, o que correspondeu a 1,1\% da carga horária total do curso inteiro. Cinco dos seis professores $(83,3 \%$ ) apresentaram pelo menos um dos componentes-chave dos PGA. A frequência de apresentação dos componentes-chave dos PGA foi de: especificidade do tratamento (5/6 - 83,3\%), duração do tratamento (4/6 - 66,7\%), otimização do tempo de administração (4/6 - 66,7\%), possibilidade de troca de via de administração (3/6 - 50\%) e monitorização de níveis séricos (2/6 - 33,3\%). Quatro dos seis docentes (66,7\%) utilizaram combinações de metodologia de ensino, as quais foram reportadas nas seguintes frequências: aulas tradicionais (5/6 - 83,3\%), estudos de casos clínicos (4/6 - 66,7\%) e aprendizado baseado em problemas (4/6 - 66,7\%). Cinquenta por cento dos docentes utilizaram combinações de avaliações para assimilação do conteúdo, e as mais utilizadas foram: respostas dissertativas curtas (3/6), casos clínicos (2/6), perguntas de múltipla escolha (2/6), resumos clínicos (1/6), seminários (1/6) e respostas dissertativas longas (1/6). Conclusões: Verificamos que os componentes-chave relativos aos PGA foram apresentados em uma escola médica do Rio de Janeiro, sendo a combinação de diferentes metodologias de ensino a principal estratégia para transmissão dos conceitos. Metade dos docentes utilizou combinação de avaliações para mensurar o conteúdo apresentado. Há necessidade de ajustes para que aspectos importantes, como troca de via de administração e monitorização de níveis séricos, sejam mais debatidos na graduação. 


\section{KEY-WORDS}

- Antimicrobial Stewardship.

- Curriculum, Education.

- Medicine, Undergraduate.

Recebido em: 22/7/19

Aceito em: 11/8/19
Introduction: Antimicrobial stewardship programs (ASP) are important tools in the effort to reduce the global issue of antimicrobial resistance. Ideally, the foundations of ASP should start to be conveyed in undergraduate medical training. Aim: To identify the presentation of ASP concepts in the curriculum of a medical school. Material and methods: A cross-sectional study conducted among medical teachers from the Universidade Federal Fluminense (UFF). The inclusion criterion was teaching ASP-related content in the undergraduate medical course. We analyzed which keyconcepts were presented, the number of hours dedicated to presentation, the teaching methodologies and the evaluation methods. Results: The study was conducted between December 2017 and January 2018 and included six of the 329 (1.8\%) teachers who taught on the Medicine course. The total hours dedicated to ASP teaching was 83; median of 3h/teacher, corresponding to $1.1 \%$ of the total class hours for the whole course. Five out of six (83.3\%) teachers present at least one key component of ASP. The frequency of key ASP concepts presented was: specificity of treatment (5/6 - 83.3\%), duration of treatment (4/6 - 66.7\%), optimization of administration time (4/6 - 66.7\%), possible switching of administration method (3/6 - 50\%) and therapeutic drug level monitoring (2/6 - 33.3\%). Four out of six $(66.7 \%)$ teachers used combinations of teaching methodologies, which were reported in the following frequency: lectures (5/6 - 83.3\%), clinical cases $(4 / 6-66.7 \%)$ and problem-based learning (4/6 - 66.7\%). Fifty percent of the teachers employed combined evaluations to measure the concepts presented and the most common used were: short dissertation questions (3/6), clinical cases (2/6), multiple choice questions (2/6), clinical summaries (1/6), seminars (1/6) and long dissertation questions (1/6). Conclusions: We verified that key ASP-related concepts were presented in a Rio de Janeiro medical school, with a combination of different methodologies constituting the main strategy to convey the concepts. Combinations of evaluations were used by half of the teachers to measure the contents presented. A requirement is observed to improve the teaching of important aspects such as when it is possible to switch the administration method and monitoring of the therapeutic drug level.

\section{INTRODUÇÃO}

A resistência antimicrobiana é um problema de saúde global, enfrentado por praticamente todos os países do mundo e reconhecido pela Organização Mundial da Saúde (OMS) como uma ameaça a ser enfrentada pelas nações ${ }^{1}$. Em termos de importância, são estimadas cerca de 700 mil mortes decorrentes de infecções por microrganismos multirresistentes e cerca de 10 milhões de óbitos anuais após 2050 caso não haja reversão desse quadro ${ }^{2}$.

Os programas de gestão de antimicrobianos (PGA) foram desenvolvidos nas últimas duas décadas com o intuito de oferecer uma estratégia de combate a este relevante problema ${ }^{3}$. Esta abordagem inclui a criação de planos nacionais direcionados contra a resistência antimicrobiana e propriamente a efetivação das ações e componentes-chave de forma local, nas instituições de assistência à saúde ${ }^{4}$.

Em dezembro de 2017, a Agência Nacional de Vigilância Sanitária publicou as diretrizes nacionais para elaboração de programas de gerenciamento do uso de antimicrobianos em serviços de saúde. Os seguintes itens foram definidos como elementos essenciais à sua criação: apoio da alta direção do hospital, definição de responsabilidades de todos os profissionais envolvidos, educação, desenvolvimento de ações para melhorar a prescrição de antimicrobianos, monitoramento do programa e divulgação de resultados ${ }^{5}$.

Apesar de as diretrizes estabelecerem as bases para a criação dos PGA, ainda há necessidade da abordagem na graduação médica dos conceitos de gestão de antimicrobianos, incluindo os principais mecanismos e formas de controle do processo de resistência antimicrobiana. O melhor entendimento destes conceitos por parte dos futuros prescritores poderia influenciar positivamente a redução do consumo e da resistência, bem como contribuir para a prescrição destes medicamentos quando estritamente recomendados.

A percepção de que o uso de antimicrobianos de amplo espectro poderia contribuir para o aumento da resistência an- 
timicrobiana foi bem reconhecida pelos estudantes de Medicina em um estudo realizado em três escolas médicas da Tailândia com 636 participantes. No entanto, o conhecimento dos mecanismos de resistência antimicrobiana, do uso apropriado de antimicrobianos e do controle de infecção foi limitado, sendo que somente $50 \%$ identificaram a presença de um PGA ou programa de controle de infecção em suas unidades ${ }^{6}$. Já Castro-Sanchez et al. ${ }^{7}$, em estudo transversal realizado em 112 cursos de graduação da saúde do Reino Unido, identificaram que 69,5\% das 24 faculdades de Medicina selecionadas ensinavam todos os princípios de gestão de antimicrobianos.

Diversas iniciativas educativas e de treinamento para profissionais da saúde têm sido realizadas com o intuito de promover um melhor conhecimento da resistência antimicrobiana. Van Katwyk et al. ${ }^{8}$ mapearam recentemente 94 iniciativas educacionais relacionadas à resistência antimicrobiana e PGA, tendo detectado uma variedade de recursos educacionais, como: cursos, workshops, conferências, guidelines, materiais de consultas públicas e websites. No entanto, nenhuma das iniciativas aconteceu na América do Sul, e em somente 4 das 94 $(4,3 \%)$ propostas o público-alvo foi o estudante de graduação em Medicina.

Levando-se em conta que as bases e conceitos dos mecanismos de resistência antimicrobiana e uso racional dos fármacos disponíveis para o tratamento adequado de infecções ocorrem na graduação médica e que estes fatores podem ser preditores de sucesso para PGA bem-sucedidos, realizamos este estudo com o objetivo de identificar se conceitos relativos à gestão de antimicrobianos são apresentados no currículo de graduação de uma escola médica.

\section{MATERIAL E MÉTODOS}

\section{Tipo de estudo e cenário}

Realizamos um estudo do tipo observacional transversal, tendo como população professores do curso de Medicina da Universidade Federal Fluminense (UFF). A UFF é uma universidade pública do Estado do Rio de Janeiro, localizada na cidade de Niterói e cujo curso de Medicina foi criado em 1925. As turmas são compostas em média por 90 alunos por semestre. $\mathrm{O}$ curso é efetivado no mínimo em 12 períodos ou no máximo em 18 períodos semestrais, com carga horária atual de 7.200 horas.

O período de estudo foi compreendido entre dezembro de 2017 e janeiro de 2018.

\section{Critérios de inclusão}

Foram incluídos no estudo professores que tinham como atribuições coordenar disciplina ou ministrar aula sobre gestão de antimicrobianos para alunos do curso de Medicina da UFF, de forma específica para alunos do curso de Medicina ou em conjunto com alunos da graduação de outros cursos da saúde da UFF.

\section{Critério de exclusão}

Foram excluídos da análise os professores que tinham como atribuições coordenar disciplina ou ministrar aula a respeito de gestão de antimicrobianos somente para alunos dos cursos de Nutrição, Biomedicina, Odontologia ou Serviço Social da UFF.

\section{Variáveis de análise}

Foram selecionadas as seguintes variáveis para análise: inclusão do tema no currículo, apresentação formal de componentes-chave dos PGA, tempo destinado à apresentação dos componentes de PGA, categoria profissional dos responsáveis pela apresentação dos conteúdos, formas de transmissão do assunto, estratégias e avaliação do conteúdo.

\section{Instrumento de coleta de dados}

Foi utilizado como referência o questionário aplicado por Castro-Sanchez et al. ${ }^{7}$ para avaliação semelhante realizada em escolas de saúde do Reino Unido, adaptado para o português. O formulário, que continha oito questões objetivas, foi preenchido pelos professores de forma presencial ou respondido por correio eletrônico.

\section{Análise dos dados}

Realizamos uma análise descritiva inicial sobre os dados encontrados, tabulados em planilha de Excel. Não foi realizada análise estatística.

\section{Aspectos éticos}

O estudo foi cadastrado na Plataforma Brasil sob o n CAEE 79092017.3.0000.5243 e aprovado pelo Comitê de Ética da UFF, conforme o Parecer no 2.545.967 de 15/03/2018.

\section{RESULTADOS}

No momento da pesquisa, 329 professores ministravam aulas para o curso de Medicina, de forma específica para o curso ou em conjunto com outros cursos de graduação. Deste total, somente seis preencheram os critérios de inclusão para análise (1,8\% do total de professores vinculados ao curso), sendo quatro médicos $(66,7 \%)$, um veterinário $(16,7 \%)$ e um biomédico $(16,7 \%)$.

A principal forma de transmissão dos conteúdos foi por meio de aula presencial, relatada por todos os seis professores $(100 \%)$. Não houve relato de aula ministrada por meio de en- 
sino à distância ou remoto. Os conteúdos foram apresentados por todos os seis docentes $(100 \%)$, de forma específica para os alunos de Medicina, sem participação de outros alunos do curso da graduação em saúde.

Quatro dos seis professores informaram que o tema gerenciamento de antimicrobianos foi incluído nas apresentações, embora cinco dos seis $(83,3 \%)$ docentes tenham apresentado ao menos um dos grandes componentes de PGA. O período de tempo total destinado ao assunto foi de 83 horas (variando de 1-60 horas), com média de 16,6 horas/docente e mediana de 3 horas, representando 1,1\% do total da carga horária do curso de Medicina. A Tabela 1 apresenta a frequência de apresentação de componentes-chave dos PGA.

\begin{tabular}{|c|c|c|}
\hline \multicolumn{3}{|c|}{$\begin{array}{l}\text { TABELA } 1 \\
\text { Frequência de apresentação de componentes- } \\
\text { chave de programas de gerenciamento de } \\
\text { antimicrobianos no curso de Medicina da UFF }\end{array}$} \\
\hline Componentes & $\begin{array}{l}\text { Frequência de apresentação/ } \\
\text { total de docentes }\end{array}$ & $\%$ \\
\hline $\begin{array}{l}\text { Especificidade do } \\
\text { tratamento antimicrobiano }\end{array}$ & $5 / 6$ & 83,3 \\
\hline Duração do tratamento & $4 / 6$ & 66,7 \\
\hline $\begin{array}{l}\text { Otimização do tempo de } \\
\text { administração }\end{array}$ & $4 / 6$ & 66,7 \\
\hline $\begin{array}{l}\text { Possibilidade de troca de } \\
\text { via de administração }\end{array}$ & $3 / 6$ & 50 \\
\hline $\begin{array}{l}\text { Monitorização de níveis } \\
\text { séricos }\end{array}$ & $2 / 6$ & 33,3 \\
\hline
\end{tabular}

As metodologias utilizadas pelos docentes para a transmissão dos conteúdos relativos ao PGA são apresentadas na Tabela 2. Quatro dos seis docentes selecionados transmitiram o conteúdo utilizando combinação de metodologias, enquanto os outros dois professores apresentaram o conteúdo usando unicamente aulas presenciais.

\begin{tabular}{|c|c|c|}
\hline \multicolumn{3}{|c|}{$\begin{array}{c}\text { TABELA } 2 \\
\text { Metodologias utilizadas pelos docentes da } \\
\text { Universidade Federal Fluminense para a transmissão } \\
\text { dos conteúdos referentes ao gerenciamento de } \\
\text { antimicrobianos para alunos de Medicina }\end{array}$} \\
\hline Metodologias & $\begin{array}{l}\text { Frequência de apresentação/ } \\
\text { total de docentes }\end{array}$ & $\%$ \\
\hline Aulas tradicionais formais & $5 / 6$ & 83,3 \\
\hline Estudo de casos clínicos & $4 / 6$ & 66,7 \\
\hline $\begin{array}{l}\text { Aprendizado baseado em } \\
\text { problemas }\end{array}$ & $4 / 6$ & 66,7 \\
\hline Seminários & $3 / 6$ & 50 \\
\hline Cenários reais & $2 / 6$ & 33,3 \\
\hline Portfólio & $0 / 6$ & 0 \\
\hline
\end{tabular}

Quanto à avaliação utilizada para verificar a assimilação do conteúdo a respeito de gerenciamento de antimicrobianos, foram utilizadas as seguintes ferramentas: respostas dissertativas curtas (3/6), casos clínicos $(2 / 6)$, perguntas de múltipla escolha (2/6), resumos clínicos $(1 / 6)$, seminários $(1 / 6)$ e respostas dissertativas longas (1/6). Três dos seis professores (50\%) utilizaram combinações de avaliações, enquanto os restantes utilizaram apenas um tipo de avaliação.

\section{DISCUSSÃO}

Os programas de gestão de antimicrobianos foram criados com o intuito de estabelecer ações efetivas contra o desenvolvimento da resistência antimicrobiana. Embora este fenômeno tenha sido relatado praticamente logo após descoberta do primeiro antibiótico, uma compilação dos mecanismos de resistência antimicrobiana existentes até a época e propostas para prevenção e redução destes agentes em hospitais foram publicadas em 1997 pela Society for Healthcare Epidemiology of America (Shea) and Infectious Diseases Society of America (IDSA $)^{9}$. A gestão de antimicrobianos foi definida posteriormente como "um conjunto de intervenções coordenadas com o objetivo de melhorar e mensurar o uso apropriado de antimicrobianos, pela promoção da seleção da melhor estratégia possível (incluindo antimicrobiano correto, na dose correta, na via de administração correta e duração adequada)"3.

A maior parte das iniciativas e propostas de intervenção dos PGA é focada no profissional de saúde já em atuação profissional nos diversos cenários de assistência, o que pode ser um fator dificultador principalmente no que se refere à necessidade de mudança de atitudes e comportamentos em relação à prescrição abusiva de antimicrobianos ${ }^{10,11}$.

Um aspecto importante debatido por diversos autores e que pode contribuir para o sucesso dos PGA é a possibilidade de melhor capacitação profissional desde a graduação médica, quando são apresentados os conceitos, mecanismos de resistência antimicrobiana e as bases para o uso racional de antimicrobiano $^{12}$.

Do nosso conhecimento, trata-se do primeiro estudo nacional a abordar o tema ensino dos conceitos de programa de gestão de antimicrobianos na graduação médica. Em nossa pesquisa, identificamos uma carga horária oficial pequena destinada à apresentação dos conceitos de PGA, correspondendo a pouco mais de $1 \%$ do total destinado ao curso, o que demonstra a necessidade de ampliar o debate do assunto. Em comparação com estudo semelhante realizado em escolas médicas do Reino Unido, a mediana de horas destinadas à apresentação dos conteúdos em nosso estudo foi menor que a mediana encontrada de 17,7 horas (variação de 8,87 h- 42,62 h) nas 13 escolas 
que mensuraram a carga horária ${ }^{7}$. A despeito da variabilidade da carga horária destinada a este tema, é possível que haja necessidade de melhor capacitação dos futuros médicos, como demonstrado por Dyar et al..$^{13}$. Esses autores avaliaram 2.085 estudantes de escolas médicas do último ano da França e Suécia a respeito de autoanálise e confiança para prescrição de antibióticos e evidenciaram que pouco mais de $63,5 \%$ dos estudantes franceses e $20,3 \%$ dos estudantes suecos consideraram a necessidade de receber mais informações sobre o uso de antibióticos.

Em relação aos componentes-chave dos PGA - como especificidade do tratamento antimicrobiano, duração do tratamento, otimização do tempo de administração, possibilidade de troca de via de administração e monitorização de níveis séricos -, verificamos que eles foram abordados em algum momento do curso, porém não por todos os professores, o que pode gerar lacunas na formação médica e posteriormente dificuldade de adesão às boas práticas no uso de antimicrobianos. Em comparação com os currículos médicos do Reino Unido, componentes-chave como minimização de prescrições desnecessárias de antimicrobianos estavam contemplados em 95,4\% dos currículos médicos, tempo de administração dos antimicrobianos em 95,4\%, monitorização de níveis séricos em 90,9\% e revisão diária da possibilidade de troca de via de administração de antimicrobianos em 81,8\%7. Estas lacunas no ensino em relação à apresentação destes componentes-chave foram demonstradas em estudo transversal realizado com estudantes australianos de Medicina do último ano. Neste trabalho, 45,8\% dos alunos relataram não estar confiantes em identificar quando o tratamento deveria ser ajustado, interrompido ou trocado por outro tratamento e $32,3 \%$ informaram falta de confiança para identificar situações em que o uso de antibióticos não era necessário ${ }^{14}$.

Outro aspecto importante do ensino dos conceitos de resistência antimicrobiana e programas de ABS é a forma de apresentação e metodologias de ensino empregadas para esta finalidade. Verificamos em nosso estudo que a maior parte dos docentes empregou combinações de metodologias, sendo as mais frequentes as aulas tradicionais formais, estudos de casos e aprendizado baseado em problemas. Segundo a percepção de estudantes espanhóis de 21 escolas médicas, as metodologias que se mostraram mais úteis no aprendizado destes tópicos foram: discussão de casos clínicos, relatada por 76,9\% dos alunos; apresentação clínica de doenças infecciosas em $76 \%$; e ensino em pequenos grupos (< 15 alunos) em $68,8 \%{ }^{15}$. Em outro estudo transversal realizado em 29 países da Europa, os métodos de ensino classificados como úteis ou muito úteis ao ensino do uso de antibióticos, conforme a avaliação dos estudantes de Medicina, foram a discussão de casos clínicos em $87,7 \%$ dos relatos e discussão em pequenos grupos em $83,9 \%{ }^{16}$.
Além da inclusão do tema nos currículos de Medicina e da aplicação de metodologias de ensino que sejam úteis e atrativas para os alunos, ainda há a necessidade de verificar se o conteúdo apresentado durante a graduação resultou efetivamente em boas práticas de uso judicioso de antimicrobianos e prevenção da resistência. Recente trabalho publicado por Silverberg et al. ${ }^{12}$ teve como objetivo rever a literatura sobre gestão de antimicrobianos na graduação e pós-graduação médica para determinar quais intervenções implementadas foram efetivas. Entre os 48 artigos incluídos na análise, nenhum deles avaliou o impacto das intervenções realizadas em estudantes de Medicina no comportamento a respeito de prescrição antimicrobiana após a licenciatura.

Nosso estudo apresenta algumas limitações, como, por exemplo, ter incluído apenas uma escola médica, o que pode refletir uma realidade regional. Isto, no entanto, não invalida o fornecimento de subsídios importantes para avaliações posteriores em outros cursos de Medicina e confirmação dos achados. É possível também que conteúdos a respeito dos PGA e resistência antimicrobiana tenham sido apresentados aos alunos de forma isolada por docentes ou profissionais de saúde do ensino prático no currículo "não formal".

Concluímos que o currículo do curso de Medicina da Universidade Federal Fluminense apresenta conceitos relativos e necessários a um programa de gestão de antimicrobianos, sendo a combinação de diferentes metodologias de ensino a principal estratégia para transmissão dos conceitos. Metade dos docentes utilizou uma combinação de avaliações para mensurar o conteúdo apresentado. É preciso ainda de estimular maior discussão, principalmente, sobre tópicos como troca de via de administração e monitorização de níveis séricos.

\section{REFERÊNCIAS}

1. World Health Organization. Ten threats to global health in 2019. Disponível em https://www.who.int/emergencies/ ten-threats-to-global-health-in-2019. Acesso em 21/03/2019.

2. O'Neill J. Review on AMR. Antimicrobial resistance: tackling a crisis for the health and wealth of nations. December, 2014. Disponível em https://amr-review.org/sites/ default / files / AMR\%20Review\%20Paper\%20-\%20Tackling $\% 20$ a $\% 20$ crisis $\% 20$ for $\% 20$ the $\% 20$ health $\% 20$ and $\% 20$ wealth\%20of\%20nations_1.pdf. Acesso em 21/03/2019.

3. Dellit TH, Owens RC, McGowan JE, Gerding DN, Weinstein RA, Burke JP, et al. Infectious Diseases Society of America and the Society for Healthcare Epidemiology of America guidelines for developing an institutional program to enhance antimicrobial stewardship. Clin Infect Dis 2007; 44: 159-77 
4. World Health Organization, Food and Agriculture Organization of the United Nations, World Organisation for Animal Health. Antimicrobial resistance: a manual for developing national action plans. 2016. Disponível em https:/ /apps.who.int/iris/handle/10665/204470. Acesso em 21/03/2019.

5. Diretriz Nacional para Elaboração de Programa de Gerenciamento do Uso de Antimicrobianos em Serviços de Saúde. Agência Nacional de Vigilância Sanitária, Brasil 2017. Disponível em http://portal.anvisa.gov.br/documents /33852/271855/Diretriz+Nacional+para+Elabora $\%$ C3\%A7\%C3\%A3o+de+Programa+de+Gerenciamento+ $\mathrm{do}+$ Uso+de+Antimicrobianos+em+Servi\%C3\%A7os+de +Sa\%C3\%BAde/667979c2-7edc-411b-a7e0-49a6448880d4. Acesso em 21/03/2019.

6. Chuenchom N, Thamlikitkul V, Chaiwarith R, Deoisares R, Rattanaumpawan P. Perception, Attitude, and Knowledge Regarding Antimicrobial Resistance, Appropriate Antimicrobial Use, and Infection Control Among Future Medical Practitioners: A Multicenter Study.Infect Control Hosp Epidemiol. 2016 May;37(5):603-5.

7. Castro-Sánchez E, Drumright LN, Gharbi M, Farrell S, Holmes AH. (2016) Mapping Antimicrobial Stewardship in Undergraduate Medical, Dental, Pharmacy, Nursing and Veterinary Education in the United Kingdom. PLoS ONE $2016 ; 11: e 0150056$

8. Van Katwyk SR, Jones SL, Hoffman SJ. Mapping educational opportunities for healthcare workers on antimicrobial resistance and stewardship around the world. Hum Resour Health. 2018 Feb 5;16(1):9.

9. Shlaes DM, Gerding DN, John JF Jr, Craig WA, Bornstein DL, Duncan RA, et al. Society for Healthcare Epidemiology of America and Infectious Diseases Society of America Joint Committee on the Prevention of Antimicrobial Resistance: Guidelines for the Prevention of Antimicrobial Resistance in Hospitals. Clin Infect Dis. 1997 Sep;25(3):584-99.

10. Rzewuska M, Charani E, Clarkson JE, Davey PG, Duncan EM, Francis JJ, et al. Joint Programming Initiative on Antimicrobial Resistance (JPIAMR) Working Group on Behavioural Approaches to Antibiotic Stewardship Programs. Prioritizing research areas for antibiotic stewardship programmes in hospitals: a behavioural perspective consensus paper.Clin Microbiol Infect. 2019 Feb;25(2):163-168.

11. Krockow EM, Colman AM, Chattoe-Brown E, Jenkins DR, Perera N, Mehtar S, et al. Balancing the risks to individual and society: a systematic review and synthesis of qualitative research on antibiotic prescribing behaviour in hospitals.J Hosp Infect. 2018 Aug 9. pii: S0195-6701(18)30418-3. doi: 10.1016/j.jhin.2018.08.007. [Epub ahead of print]

12. Silverberg SL, Zannella VE, Countryman D, Ayala AP, Lenton E, Friesen F, et al. A review of antimicrobial stewardship training in medical education.Int J Med Educ. 2017 Oct 12;8:353-374.

13. Dyar OJ, Lund M, Lindsjö C, Lundborg CS, Pulcini C. French-Swedish Student-PREPARE ESGAP working group. Preparedness to prescribe antibiotics responsibly: a comparison between final year medical students in France and Sweden. Eur J Clin Microbiol Infect Dis. 2019 Apr;38(4):711-717.

14. Weier N, Thursky K, Zaidi STR. Antimicrobial knowledge and confidence amongst final year medical students in Australia.PLoS One. 2017 Aug 3;12(8):e0182460

15. Sanchez-Fabra D, Dyar OJ, del Pozoc JL, Amiguet JÁ, Colmenero JD, Fariñas MC, et al, on behalf of ESGAP (ESCMID Study Group for Antimicrobial Stewardship) Perspective of Spanish medical students regarding undergraduate education in infectious diseases, bacterial resistance and antibiotic use. EnfermInfecc Microbiol Clin. 2019;37(1):25-30

16. Dyar OJ, Nathwani D, Monnet DL, Gyssens IC, Lundborg CS, Pulcini C, on behalf of the ESGAP Student-PREPARE Working Group. Do medical students feel prepared to prescribe antibiotics responsibly? Results from a cross-sectional survey in 29 European countries. J AntimicrobChemother 2018; 73: 2236-2242

\section{CONTRIBUIÇÃO DOS AUTORES}

André Ricardo Araujo da Silva: concepção do artigo, redação do artigo, revisão final; Igor Melo de Almeida: coleta de dados, revisão bibliográfica, revisão final; Rafael Carvalho Sacre: coleta de dados, revisão bibliográfica, revisão do abstract, revisão final; Cristina Letícia Passos de Souza: coleta de dados, revisão bibliográfica, revisão final.

\section{CONFLITO DE INTERESSES}

Sem conflito de interesses.

\section{ENDEREÇO PARA CORRESPONDÊNCIA}

André Ricardo Araujo da Silva

Rua Professor Gabizo 252, bloco 6, apto 501

Maracanã - Rio de Janeiro

CEP 20271-062 - RJ

E-mail: aricardo@id.uff.br 